\title{
Individual Participant Symptom Responses to Intra- Articular Lorecivivint in Knee Osteoarthritis: Post Hoc Analysis of a Phase 2B Trial
}

\author{
Jeyanesh R. S. Tambiah (D) · Sarah Kennedy • Christopher J. Swearingen • \\ Ismail Simsek · Yusuf Yazici · Jack Farr · Philip G. Conaghan
}

Received: April 15, 2021 / Accepted: May 7, 2021 / Published online: June 8, 2021

(C) The Author(s) 2021

\section{ABSTRACT}

Introduction: Established thresholds for patient-reported outcomes (PROs) provide clinically relevant responder data from trials. Lorecivivint (LOR) is an intra-articular (IA) therapy in development for knee osteoarthritis (OA). A post hoc analysis from a phase $2 \mathrm{~b}$ trial (NCT03122860) determined proportions of LOR responders.

Methods: A 24-week, randomized trial of $0.07 \mathrm{mg}$ LOR demonstrated PRO improvements compared with $\mathrm{PBO}$ in moderate-to-severe knee OA participants. Participants treated with LOR and $\quad \mathrm{PBO}$ achieving 30\%/50\%/70\%

Supplementary Information The online version contains supplementary material available at https:// doi.org/10.1007/s40744-021-00316-w.

J. R. S. Tambiah (ه) · S. Kennedy •

C. J. Swearingen · I. Simsek · Y. Yazici

Biosplice Therapeutics, Inc., 9360 Towne Centre Dr, San Diego, CA 92121, USA

e-mail: jeymi.tambiah@biosplice.com

Y. Yazici

New York University School of Medicine, New York, NY, USA

J. Farr

OrthoIndy Hospital, Indianapolis, IN, USA

P. G. Conaghan

Leeds Institute of Rheumatic and Musculoskeletal

Medicine, University of Leeds, and NIHR Leeds

Biomedical Research Centre, Leeds, UK improvements at weeks 12 and 24 in Pain Numeric Rating Scale (NRS), WOMAC Pain/ Function subscales, Patient Global Assessment (PtGA), and OMERACT-OARSI responder criteria were determined. Odds ratios (ORs) and 95\% confidence intervals [CIs] were compared with PBO.

Results: There were 115 and 116 participants in the LOR and PBO groups, respectively. For Pain NRS, LOR increased ORs of achieving 30\% [week 12 , OR $=2.47(1.45,4.19), P<0.001$; week 24 , OR $=2.37(1.40,4.02), P<0.01]$ and 50\% [week 24 , OR $=1.89(1.11,3.23), P<0.05]$ improvements over baseline. For WOMAC Pain, LOR increased ORs of achieving 30\% [week 24, OR $=1.79(1.06,3.01), P<0.05$ ] and 50\% [week $12, \mathrm{OR}=1.79(1.06,3.03), P<0.05$; week 24 , OR $=1.73(1.02,2.93), P<0.05]$ improvements. For WOMAC Function, LOR increased ORs of achieving 30\% [week 12, OR = $1.85(1.10,3.12)$, $P<0.05$; week 24 , OR $=1.93 \quad(1.14,3.26)$, $P<0.05]$ improvements. For PtGA, LOR increased ORs of achieving 50\% [week 12, $\mathrm{OR}=2.28(1.25,4.16), P<0.01]$ improvements. LOR produced numerical increases at the $70 \%$ threshold. LOR increased ORs of achieving OMERACT-OARSI responses [week 12, OR = $2.21(1.29,3.78) ; P<0.01$; week $24, \mathrm{OR}=2.57$ (1.49, 4.43), $P<0.001]$ and strict responses [week 12, OR $=2.13(1.26,3.61), P<0.01$; week $24, \mathrm{OR}=2.05(1.21,3.47), P<0.01]$.

Conclusions: LOR (0.07 mg) demonstrated improved PRO threshold responses across single 
and composite measures of pain, function, and patient global assessment compared with PBO, with benefits sustained to 24 weeks.

\section{PLAIN LANGUAGE SUMMARY}

Lorecivivint (LOR) is a new injectable medicine being studied as a treatment for knee osteoarthritis (OA). An early (phase 2b) trial found participants with moderate-to-severe knee OA receiving LOR on average reported improved pain, function, and reduced impact of OA symptoms over 24 weeks compared with placebo. To consider how likely individuals were to respond to treatment, this study analyzed how many participants per group achieved different percentage levels of symptom improvement. Participants were given a single LOR or placebo injection into their most painful (target) knee at trial initiation. Participants reported their target knee status from day 1 (baseline) to week 24 using pain and function questionnaires. We analyzed the number of participants given $0.07 \mathrm{mg}$ LOR and placebo whose symptom scores improved by 30,50 , and
$70 \%$ over baseline scores at weeks 12 and 24 . Results showed that $0.07 \mathrm{mg}$ LOR treatment produced a higher likelihood beyond chance at week 12 of achieving a 30\% improvement in some pain and function scores and a 50\% improvement in other symptom scores compared with placebo. Similar 30\% and 50\% symptom score improvements were found at week 24. More complex scores, combining individual symptom scores into single index measures, also showed improvements beyond chance for $0.07 \mathrm{mg}$ LOR from baseline compared with placebo at weeks 12 and 24. Thus, more participants with knee OA who were treated with $0.07 \mathrm{mg}$ LOR demonstrated longlasting, meaningful improvements in pain and function compared to those given placebo.

Keywords: Alternative splicing; CLK2; DYRK1A; OMERACT-OARSI; Patient-reported outcomes; Wnt signaling pathway 
Infographic:

Individual Participant Symptom Responses to Intra-Articular Lorecivivint in Knee Osteoarthritis: Post Hoc Analysis of a Phase 2B Trial

Cite This Article As: Tambiah J, Kennedy S, Swearingen C,

https://doiorg I Yazici Y, Farr J, Conaghan P

WORLDWIDE PREVALENCE OF KNEE

OSTEOARTHRITIS IS ALMOST 4\%

\section{Purpose}

Determine the clinically

important pain and function

responses to lorecivivint in participants with knee OA

\section{Key Findings}

Participants receiving lorecivivint were statistically more likely to achieve at least a $30 \%$ improvement in pain compared to placebo at 12 and 24 weeks

\section{Lorecivivint}

1st in class investigational intra-articular injection that is intra-articular injection that is an inhibitor of CLK2 and and inflammatory pathways

\section{Pain Responder Rates}

\section{Lorecivivint $64.3 \%$}

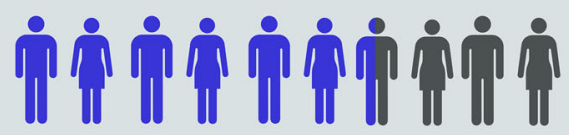

Placebo

$42.2 \%$

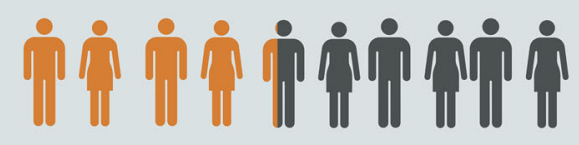

LORECIVIVINT-TREATED

PARTICIPANTS EXPERIENCED CLINICALLY

MEANINGFUL IMPROVEMENTS IN PAIN AND FUNCTION
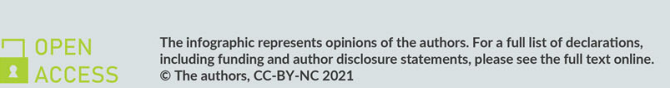

$\triangle$ Adis PEER-REVEWED
INFOGRAPHIC 


\section{Key Summary Points}

\section{Why carry out this study?}

Lorecivivint (LOR) is being studied as a potential new injectable medicine treatment for knee osteoarthritis (OA).

This analysis was conducted to determine the proportion of subjects treated by intraarticular injection with $0.07 \mathrm{mg}$ LOR or placebo who achieved a clinically meaningful response according to individual or combined self-rated pain or function symptom scores.

\section{What was learned from the study?}

We found that $0.07 \mathrm{mg}$ LOR treatment produced a higher likelihood (beyond chance) at week 12 of achieving $30 \%$ or $50 \%$ improvements over baseline in some pain and function scores compared with placebo, with similar symptom score improvements at week 24 .

When tested using the combined symptom score criteria, $0.07 \mathrm{mg}$ LOR treatment also showed improvements beyond chance at weeks 12 and 24 when compared with placebo.

This study showed that more participants with knee OA who were treated with LOR demonstrated durable, clinically meaningful improvements in pain and function than did those given a placebo.

\section{DIGITAL FEATURES}

This article is published with digital features, including a summary slide and infographic, to facilitate understanding of the article. To view digital features for this article go to https://doi. org/10.6084/m9.figshare.14541393.

\section{INTRODUCTION}

Knee osteoarthritis (OA) is a prevalent $(\sim 3.8 \%$ globally) joint condition characterized by chronic pain and joint degeneration due to cartilage degradation and osteophyte formation. This leads to impaired function and reduced quality of life for patients [1]. The lack of treatments that modify the underlying pathophysiology of the disease, limited efficacy, and/or safety concerns associated with current treatments represent unmet needs for new knee OA drugs to address.

Patient-reported outcomes (PROs) such as the Pain Numeric Rating Scale (NRS), the Western Ontario and McMaster Universities Arthritis Index (WOMAC) scales, and the Patient Global Assessment (PtGA), are sensitive and specific measures commonly utilized for clinical trials in knee OA. PROs are validated questionnaires that capture patient views of their health status, and are often presented within clinical trials as group mean changes from baseline over time. To gain a patient-level perspective on a test drug's efficacy, results can be evaluated according to discrete percentage thresholds, which can identify proportions of participants achieving clinically meaningful responses $[2,3]$. This method provides additional information that could be more relevant to a treating physician and increase interpretability of the trial results [4].

Other criteria that can enhance understanding of the meaningfulness of trial data are composite responder outcomes, which can demonstrate improvements across multiple PRO domains (e.g., in both pain and function) from a patient-level perspective. An example is the Outcomes Measures in Rheumatology-Osteoarthritis Research Society International (OMERACT-OARSI) scenario D responder criteria, which describe moderate (responder) and large (strict responder) responses that require both absolute and relative improvements in at least two of WOMAC Pain, WOMAC Function, and PtGA outcome scores [5].

Lorecivivint (LOR) is a small-molecule drug that is currently in development as a potential intra-articular (IA) therapy for knee OA. LOR 
inhibits CLK2 and DYRK1A, two intranuclear kinases that modulate the Wnt signaling pathway and inflammation and has demonstrated improvements in pain, function, and potentially joint structure in knee OA subjects $[6,7]$. In a 24-week, randomized, double-blind, controlled phase 2b trial (NCT03122860), LOR demonstrated significant group-level improvements from baseline in pain and function PROs (Pain NRS, WOMAC Pain and Function subscales, and PtGA) compared with placebo in participants with moderate-to-severe knee OA (primary data reported elsewhere [8]).

To evaluate participants' threshold improvements in PROs over time, a post hoc analysis of data from this trial was performed. A dose-finding analysis within the phase $2 \mathrm{~b}$ trial identified $0.07 \mathrm{mg}$ LOR as the lowest effective dose [8]. As this dose was selected for subsequent phase 3 studies, we analyzed data for the $0.07 \mathrm{mg}$ LOR dose and vehicle placebo (PBO) injections at week 12 and week 24 . Results are reported for thresholds of 30, 50, and 70\% improvement from baseline responses, as well as with OMERACT-OARSI criteria.

\section{METHODS}

\section{Study Design}

The clinical trial in which these data were collected was a 24 -week, phase $2 \mathrm{~b}$, multicenter, randomized, double-blind, PBO-controlled, parallel-group clinical trial of LOR injected into the target knee joint of participants with moderately to severely symptomatic OA. PRO endpoints included changes of target knee pain and function (Pain NRS, WOMAC Pain and Function subscales, and PtGA) from baseline to week 24 for LOR compared with vehicle PBO. A detailed description of the trial has been published [8].

The trial was conducted in accordance with the Declaration of Helsinki, the International Council for Harmonisation Good Clinical Practice Guidelines, and applicable regulations. All sites conducted the trial under institutional review board approval. All participants provided written informed consent prior to participating in any study-related procedures.

\section{Participants}

Eligible participants were adults aged 40-80 years with a diagnosis of primary femorotibial OA in the target knee according to clinical and radiographic American College of Rheumatology criteria; participants were otherwise expected to be in general good health and ambulatory with Kellgren-Lawrence (KL) grade 2 or 3 disease in the target knee. Participants were excluded if receiving any pharmacological OA treatments besides NSAIDs or acetaminophen, but there were no restrictions on NSAID/acetaminophen use before or during the trial period.

Participants had knee OA pain for at least 26 weeks prior to initial screening. In the knee designated for injection (target knee), participants must have reported a weekly average of daily Pain NRS intensity scores between 4 and 8 (inclusive) on the 11-point [0-10] scale from $\geq 4$ measurements during the week preceding treatment initiation, with a daily average NRS intensity score $<4$ in the non-target knee. In addition, participants were required to have a WOMAC (version 3.1) total score of 96-192 [0-240] for the target knee at baseline regardless of whether the subject was on oral NSAID or acetaminophen treatment.

\section{Study Protocol}

Eligible participants were randomized into $0.03 \mathrm{mg}, 0.07 \mathrm{mg}, 0.15 \mathrm{mg}$, and $0.23 \mathrm{mg}$ LOR dose groups and vehicle $\mathrm{PBO}$ and sham (dry needle) control groups. All participants received a single $2.0 \mathrm{ml}$ IA injection into the target knee on day 1 . Study investigators, research personnel, and participants were blinded to group assignment, whereas unblinded personnel prepared medication and performed injections.

Subject characteristics, medical history, and body mass index (BMI) were collected at screening. Participants were required to complete an electronic diary (ERT, New York, NY) to assess daily Pain NRS, as well as monthly 
completion of the WOMAC and PtGA. Analyses were conducted with the full analysis set (FAS), which included all participants who were randomized and treated; results of the primary analysis are reported elsewhere [8].

\section{Responder Analyses}

As a post hoc analysis, the proportions of participants in the LOR $0.07 \mathrm{mg}$ and $\mathrm{PBO}$ groups whose PRO responses met a 30,50 , or $70 \%$ improvement threshold over baseline were determined at weeks 12 and 24 . These thresholds were chosen based on published analyses and a number of studies previously reporting these same cut-offs [2, 5, 9-13]. Additionally, the proportions of participants whose responses met the large OMERACT-OARSI response (50\% relative and 20-point absolute [scaled 0-100] improvement, referred to as "strict responder") criteria in either Pain NRS or WOMAC Function or moderate OMERACT-OARSI response (20\% relative and 10-point absolute [scaled 0-100] improvement, referred to as "responder") in 2 of 3 of Pain NRS, WOMAC Function, and PtGA domains were assessed [5]. The proportions of participants in the $0.07 \mathrm{mg}$ LOR treatment group meeting each response threshold at weeks 12 and 24 were compared groupwise with the PBO group; odds ratios (OR) and 95\% confidence intervals $\left[\begin{array}{ll}95 \% & \mathrm{CI}\end{array}\right]$ of participants achieving each threshold response level with LOR compared with PBO were calculated using logistic regression on the full analysis set with non-responder imputation. Type 1 error control was employed for the primary dose-response analysis [8] but not in this post hoc response analysis.

\section{RESULTS}

Participant disposition is described in Fig. 1. One-hundred fifteen participants (mean age $59.9[ \pm 8.6]$ years, BMI $29.1[ \pm 3.6] \mathrm{kg} / \mathrm{m}^{2}$, female $57.4 \%$, KL grade $364.3 \%$ ) were randomized to the $0.07 \mathrm{mg}$ LOR group and 116 (mean age 60.1 $[ \pm 9.0]$ years, BMI $28.6[ \pm 4.3]$ $\mathrm{kg} / \mathrm{m}^{2}$, female $55.2 \%$, KL grade $362.1 \%$ ) were randomized to the PBO group. No differences in baseline subject characteristics were identified (see Supplementary Table 1 and [8]). Briefly, treatment with $0.07 \mathrm{mg}$ LOR demonstrated statistically significant group-level improvements compared with $\mathrm{PBO}$ at week 12 in Pain NRS (Supplementary Fig. 1), WOMAC Pain, and WOMAC Function. At week 24, statistically significant improvements compared with $\mathrm{PBO}$ in Pain NRS were demonstrated with $0.07 \mathrm{mg}$ LOR.

In the current analysis, $0.07 \mathrm{mg}$ LOR treatment significantly increased the odds of demonstrating a 30\% improvement response over baseline score for Pain NRS at week 12 [LOR 64.3\% responders, PBO 42.2\%, OR $=2.47$ $(1.45,4.19) ; P<0.001]$ and week 24 [LOR $61.7 \%, \quad \mathrm{PBO} 40.5 \%, \quad \mathrm{OR}=2.37 \quad(1.40,4.02)$; $P<0.01]$, and those of achieving a 50\% improvement over baseline at week 24 [LOR $47.0 \%, \quad$ PBO $31.9 \%, \quad$ OR $=1.89 \quad(1.11,3.23)$; $P<0.05]$, compared with PBO (Fig. 2a). For WOMAC Pain, the $0.07 \mathrm{mg}$ LOR treatment group demonstrated significantly increased odds of achieving a 50\% improvement response over baseline at week 12 [LOR 50.4\%, PBO $36.2 \%, \mathrm{OR}=1.73(1.02,2.93) ; P<0.05]$, and of achieving a 30\% [LOR 60.9\%, PBO 46.6\%, $\mathrm{OR}=1.79(1.06,3.03) ; P<0.05]$ and $50 \%$ [LOR $49.6 \%, \quad \mathrm{PBO} 36.2 \%, \quad \mathrm{OR}=1.73 \quad(1.02,2.93)$; $P<0.05$ ] improvement response over baseline at week 24 compared with PBO (Fig. 2b). For WOMAC Function, LOR treatment significantly increased the odds of demonstrating a 30\% improvement response over baseline at week 12 [LOR 60.9\%, PBO 45.7\%, OR = $1.85(1.10,3.12)$; $P<0.05$ ] and week 24 [LOR 63.5\%, PBO 47.4\%, $\mathrm{OR}=1.93(1.14,3.26) ; P<0.05]$ compared with PBO (Fig. 3a). LOR treatment also significantly increased the odds of demonstrating a 50\% improvement response over baseline score for PtGA at week 12 [LOR 34.8\%, PBO 19.0\%, $\mathrm{OR}=2.28(1.25,4.16) ; P<0.01]$ compared with PBO (Fig. 3b). Treatment with $0.07 \mathrm{mg}$ LOR produced numerical increases in responders achieving a 70\% threshold response with all PROs at week 12 [Pain NRS, LOR 30.4\%, PBO $22.4 \%, \mathrm{OR}=1.51(0.84,2.73)$; WOMAC Pain, LOR 29.6\%, PBO 23.3\%, OR = $1.38(0.77,2.49)$; WOMAC Function, LOR 29.6\%, PBO 23.3\%, $\mathrm{OR}=1.38(0.77,2.49) ;$ PtGA, LOR 20.9\%, PBO 


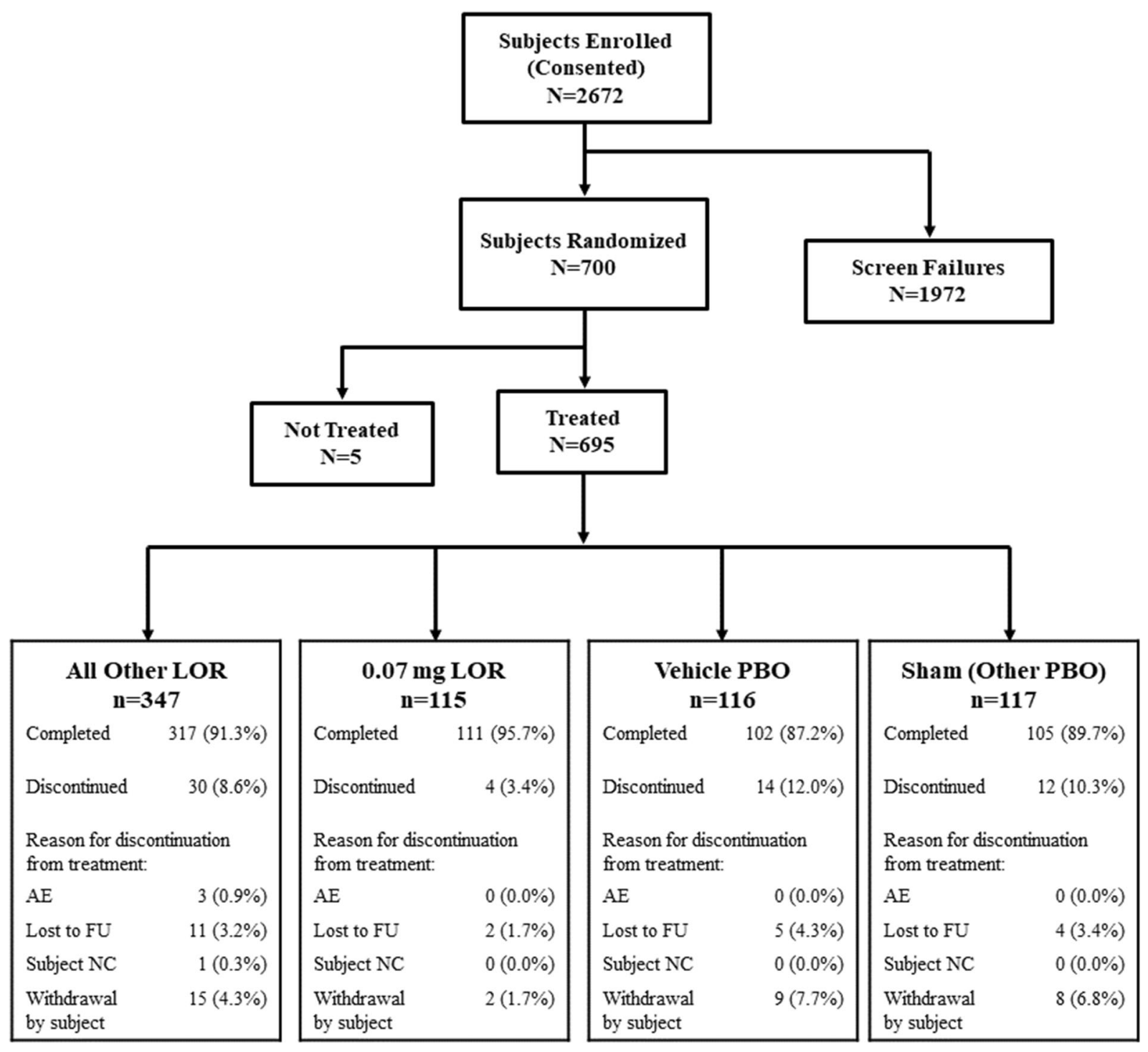

Fig. 1 Phase $2 \mathrm{~b}$ trial patient disposition diagram. The post hoc analyses in this paper were performed on the groups receiving intra-articular $0.07 \mathrm{mg}$ lorecivivint (LOR) and vehicle placebo (Vehicle PBO) injections

$13.8 \%$, OR $=1.65(0.82,3.30)]$ and week 24 [Pain NRS, LOR 29.6\%, PBO 22.4\%, OR = 1.45 $(0.80,2.63)$; WOMAC Pain, LOR 34.8\%, PBO $30.2 \%$, OR $=1.23(0.71,2.14)$; WOMAC Function, LOR 33.0\%, PBO 26.7\%, OR = $1.35(0.77$, 2.38); PtGA, LOR 21.7\%, PBO 13.8\%, OR = 1.74 $(0.87,3.46)]$. However, none of these increases reached statistical significance $(P \geq 0.05$ in all cases) compared with PBO.
When compared with $\mathrm{PBO}, 0.07 \mathrm{mg}$ LOR treatment significantly increased the proportion of OMERACT-OARSI responders [LOR 69.6\%, PBO 50.9\%, OR $=2.21 \quad(1.29,3.78)$; $P<0.01$ ] and strict responders [LOR 55.7\%, PBO 37.1\%, OR = $2.13(1.26,3.61) ; P<0.01]$ at week 12 and week 24 [responders, LOR 71.3\%, PBO 49.1\%, OR = $2.57(1.49,4.43) ; P<0.001$; strict responders, LOR 55.7\%, $\mathrm{PBO} 37.9 \%$, $\mathrm{OR}=2.05$ (1.21, 3.47); $P<0.01]$ (Fig. 4). 


\section{A}
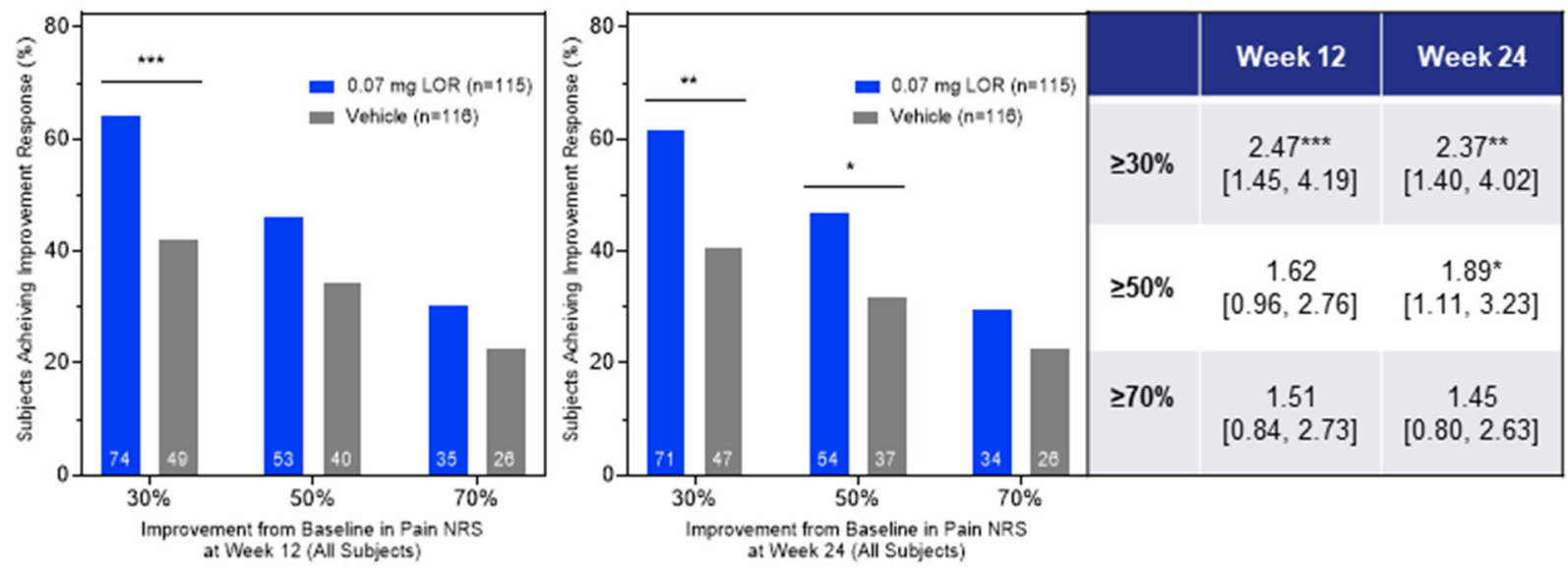

B

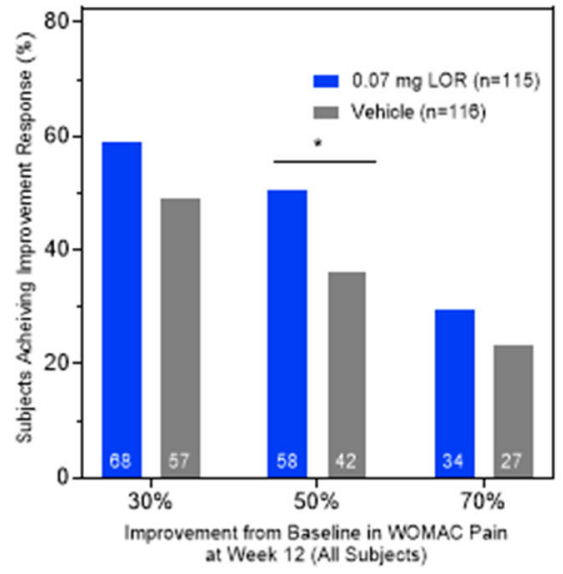

Fig. 2 Participants meeting 30, 50, and 70\% response improvement thresholds in the Pain NRS and WOMAC Pain scales. a The numbers (values at the column bases) and proportions of participants in the lorecivivint (LOR, blue) and Vehicle placebo (PBO, gray) groups meeting each threshold in Pain NRS at weeks 12 (left) and 24 (right) are shown in the bar graphs; odds ratios (ORs) and 95\% confidence intervals [95\% CIs] are provided in the

\section{DISCUSSION}

The original phase $2 \mathrm{~b}$ trial [8] showed that the $0.07 \mathrm{mg}$ LOR dose met the primary endpoint of improvement in Pain NRS at 24 weeks compared with PBO in participants with moderate- adjacent table. $\mathbf{b}$ The number and proportion of participants in the LOR and $\mathrm{PBO}$ groups meeting each threshold in WOMAC Pain at weeks 12 and 24 and accompanying ORs/95\% CIs. Statistically significant comparisons between groups are identified by asterisks $\left({ }^{*} P<0.05,{ }^{* *} P<0.01,{ }^{* * *} P<0.001\right)$; significance was determined by logistic regression with non-responder imputation

to-severe knee OA. LOR was well-tolerated in this trial. In this post hoc analysis of these data, participants receiving $0.07 \mathrm{mg}$ LOR demonstrated statistically significant improvements in the odds of meeting $30 \%$ or $50 \%$ PRO response improvements, or OMERACT-OARSI responses/ strict responses compared with $\mathrm{PBO}$ at weeks 12 

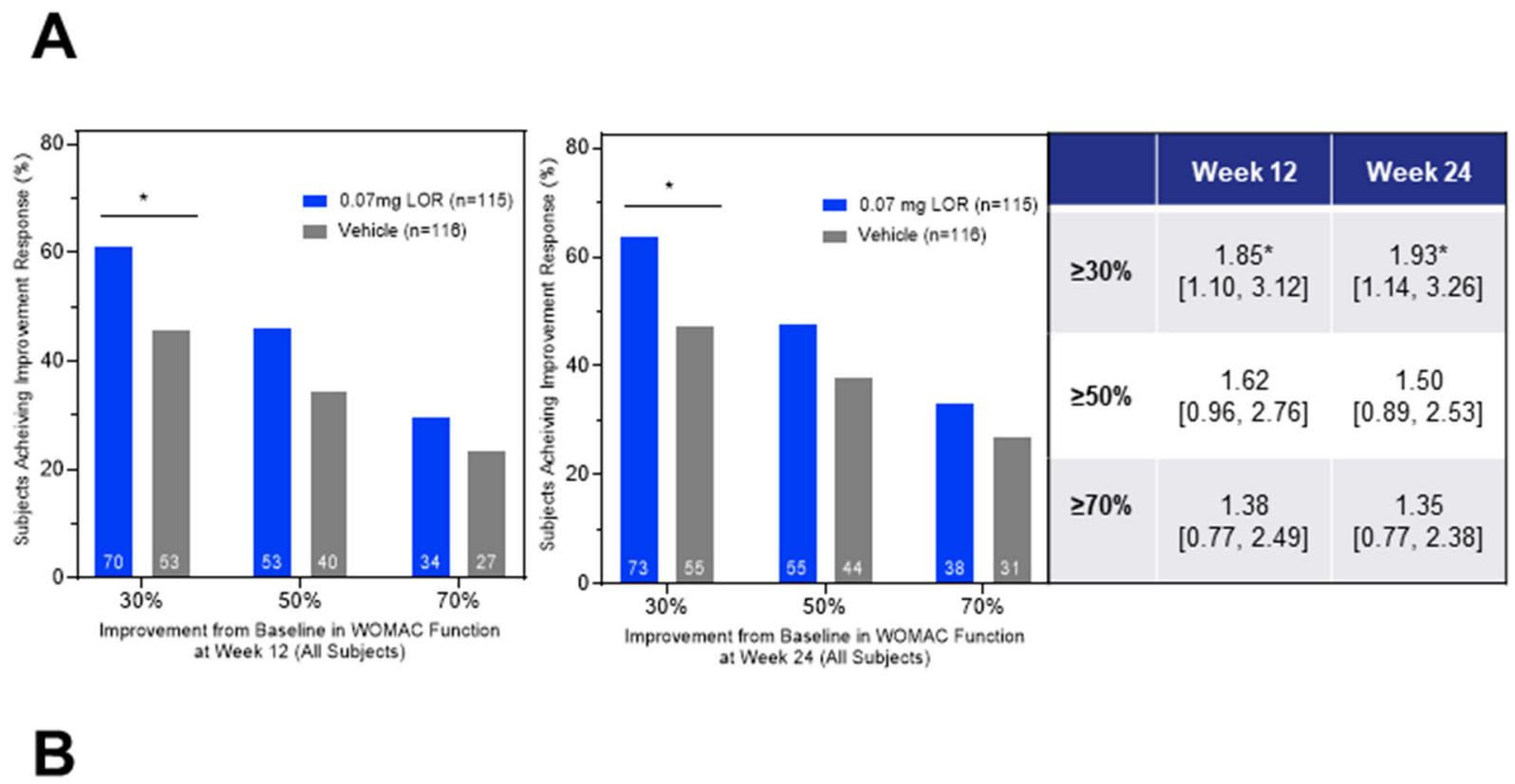

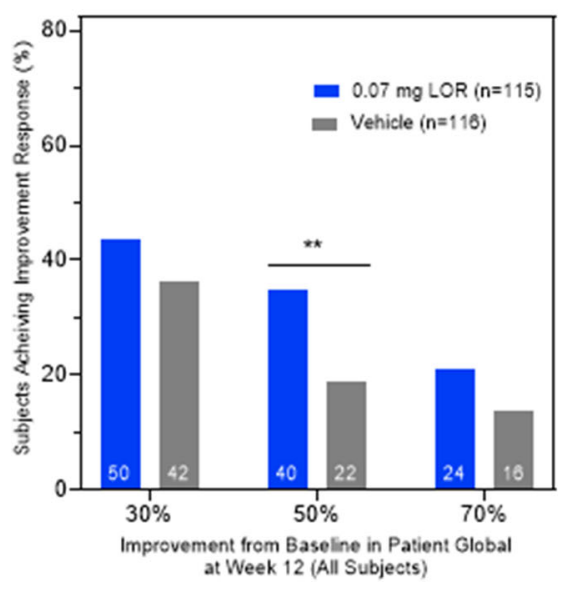

Fig. 3 Participants meeting 30, 50, and 70\% response improvement thresholds in the WOMAC Function and Patient Global (PtGA) scales. a The numbers (values at the column bases) and proportions of participants in the lorecivivint (LOR, blue) and Vehicle placebo (PBO, gray) groups meeting each threshold in WOMAC Function at weeks 12 (left) and 24 (right) are shown in the bar graphs; odds ratios (ORs) and $95 \%$ confidence intervals [95\% CIs]

and 24. Numerically, but not statistically, improved odds of meeting a 70\% threshold response were also noted.

Previously, pain thresholds have been defined as "moderate" if $\geq 30 \%$, or "substantial" if $\geq 50 \%$, PRO improvements over baseline were achieved [2]. By these definitions, LOR trial participant responder rates were $60 \%$ for moderate, and $45 \%$ for substantial improvements over baseline in Pain NRS at both weeks 12 and 24 (Fig. 2a). Similarly, for WOMAC Pain and Function subscores, a responder rate profile 

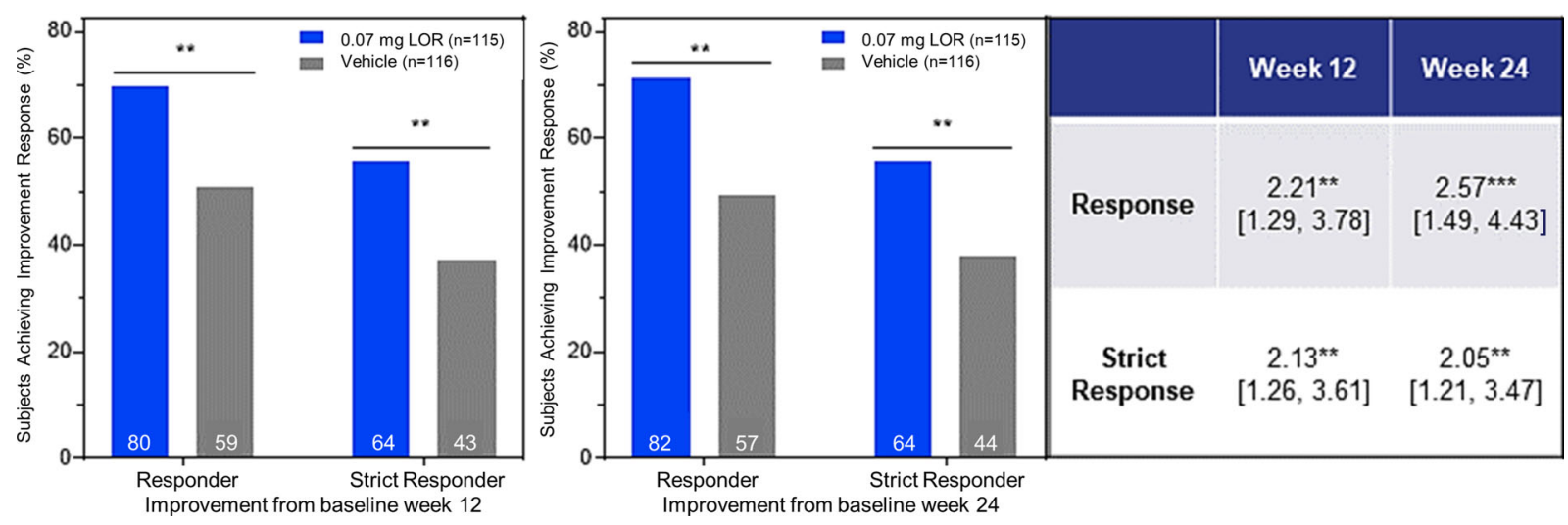

Fig. 4 Participants meeting OMERACT-OARSI responder and strict responder criteria. (Top) The number and proportion of participants in the lorecivivint (LOR, blue) and Vehicle placebo (PBO, gray) groups meeting OMERACT-OARSI responder (Response) and strict responder ("Strict" Response) at week 12 are shown in the bar graph; odds ratios (ORs) and 95\% confidence intervals (95\% CIs)

of approximately $60 \%$ for moderate and $45 \%$ for substantial threshold achievements were also observed at weeks 12 and 24 . These results therefore demonstrated that $0.07 \mathrm{mg}$ LOR treatment yielded consistent PRO responder scores, often statistically significant over PBO and sustained over 24 weeks (Figs. 2, 3). Also of note, were the percentages of $\mathrm{PBO}$ responders achieving 30/50/70\% thresholds, which appeared consistent (approximately 40-45, $30-35$, and 20-25\%, respectively) across Pain NRS and WOMAC Pain/Function scores at both timepoints. These were similar to the sustained PRO responses observed in $\mathrm{PBO}$ arms from many IA knee OA trials reporting group mean changes over time [14].

A lower responder rate profile was observed for PtGA scores (Fig. 3b), in which $40-45 \%$ of LOR participants improved by $30 \%$, and $30-35 \%$ improved by $50 \%$ at weeks 12 and 24 , respectively. LOR remained numerically higher than $\mathrm{PBO}$ at both timepoints (and statistically higher for $50 \%$ responders at week 12), with mean ORs ranging from 1.36 to 2.28 .

OMERACT-OARSI responder index criteria defines "moderate" (corresponding to the "responder" group in this analysis), and "large" (corresponding to the "strict responder" group) are provided in the adjacent table. (Bottom) The number and proportion of participants in the LOR and $\mathrm{PBO}$ groups meeting each criterion level at week 24 and accompanying ORs $/ 95 \%$ CIs. Statistically significant comparisons between groups are identified by asterisks $\left({ }^{* *} P<0.01,{ }^{* * *} P<0.001\right)$; significance was determined by logistic regression with non-responder imputation

improvements. Strict responders were a subset of responders in this analysis (Fig. 4) [5]. With this composite responder index, about $70 \%$ of $0.07 \mathrm{mg}$ LOR participants achieved a moderate response at weeks 12 and 24. Approximately $56 \%$ of the LOR-treated group achieved a large response at weeks 12 and 24 . These results were each statistically significant compared with PBO at both timepoints.

In summary, this study examined several clinically meaningful improvement thresholds, from baseline, of single-domain PROs at 12 and 24 weeks, with a greater number of $0.07 \mathrm{mg}$ LOR participants consistently achieving these thresholds compared with PBO. Analysis of composite OMERACT-OARSI responses further demonstrated that those treated with $0.07 \mathrm{mg}$ LOR also achieved improvements across multiple PRO domains over baseline and compared with PBO.

Population sample mean trends that are usually reported from randomized clinical trials show the trajectory of a treatment group's responses to a treatment over time. Threshold responses, on the other hand, give a snapshot of the proportion of trial participants achieving a clinically meaningful improvement in symptoms at an individual timepoint. The 
OMERACT-OARSI responses give a further perspective on knee OA disease symptomology, as the complex relative relationship of patient-reported OA pain, function, and well-being are combined within these scores. This is reflected in the data reported, as LOR achieved statistical significance compared with PBO for responder and strict responder scores at both week 12 and 24 timepoints, in contrast to the single domain PRO responses. However, it is important to note with threshold and responder scores, it is not possible to know if individual participants maintained, changed, or lost their responder status over the course of a trial when looking at consecutive timepoints. Therefore, analyzing these categorical participant-level improvements, together with assessing group mean changes, allows for an overall more clinically relevant interpretation of a drug's treatment benefits.

These LOR data can also be considered in the context of other OA pharmacotherapies, with the caveat that different trial designs prevent true direct comparisons being made. The LOR responder rates in this analysis were generally similar to the ranges of values published in the literature with existing, e.g., duloxetine [9-11] and emerging, e.g., anti-nerve growth factor, therapies $[12,13]$. Of note, the phase $2 b$ LOR trial design did not include a typical pre-trial oral analgesic washout, and these data presented were with participants potentially continuing their background NSAIDs and acetaminophen. Along with symptomatic benefits, the mechanism of action of LOR suggests structural improvements to the knee joint may also result from treatment [6-8]. These effects are also being investigated as part of the development program.

Limitations of this report included that this was a post hoc analysis, which was not specifically error-controlled, however characterized responses from a study that met its primary endpoint while under error control. Also the study was conducted in a selected trial population, which may limit translation of these results to a real-world clinical population.

\section{CONCLUSIONS}

In conclusion, this report summarizes a post hoc analysis of a 24 -week phase $2 \mathrm{~b}$ clinical trial in symptomatic knee OA participants who were treated with a single injection of $0.07 \mathrm{mg}$ LOR. Specifically, it demonstrated that the significant, durable treatment effects of $0.07 \mathrm{mg}$ LOR found in the primary data, were also observed in this threshold response analysis, with responders achieving meaningful single and composite PRO improvements compared with $\mathrm{PBO}$ at 12 and 24 weeks.

\section{ACKNOWLEDGEMENTS}

The authors gratefully acknowledge and thank the study participants, investigators, and associated research professionals who contributed their time and efforts to these clinical studies. The authors would also like to thank the Biosplice team for their contributions to these clinical studies. PGC is supported in part by the UK National Institute for Health Research (NIHR) Leeds Biomedical Research Centre. The views expressed are those of the authors and not necessarily those of the NHS, the NIHR, or the Department of Health.

Funding. All studies described in this report, the journal's Rapid Service Fee, and the preparation of this manuscript were funded by Biosplice Therapeutics, Inc.

Medical writing and Editorial Assistance. The authors would also like to thank Sarah Snyder for assistance in developing the infographic; Andrew Ray, Andrea Carrano, and Elizabeth Orient for critical revision of and editorial assistance with this manuscript.

Authorship. All named authors meet the International Committee of Medical Journal Editors (ICMJE) criteria for authorship for this article, take responsibility for the integrity of the work as a whole, and have given their approval for this version to be published. 
Authors' Contributions. Jeyanesh R.S. Tambiah: Study design, data acquisition, data analysis, and data interpretation; drafting, critical revision, and final approval of the manuscript Sarah Kennedy: Study design, data acquisition, data analysis, and data interpretation; drafting, critical revision, and final approval of the manuscript. Christopher J. Swearingen: Study design, data acquisition, data analysis, and data interpretation; drafting, critical revision, and final approval of the manuscript. Ismail Simsek: Study design, data acquisition, data analysis, and data interpretation; drafting, critical revision, and final approval of the manuscript. Yusuf Yazici: Study design, data acquisition, data analysis, and data interpretation; critical revision and final approval of the manuscript. Jack Farr: Data interpretation; critical revision and final approval of the manuscript. Philip G. Conaghan: Data interpretation; critical revision and final approval of the manuscript.

Disclosures. Drs. Tambiah, Kennedy, Swearingen, Simsek, and Yazici own stock or stock options in Biosplice Therapeutics. Dr. Farr received consulting and/or speaking fees from Aesculap/B. Braun, Arthrex, Cartiheal, Cook Biotech, Exactech, Moximed, Organogenesis, Regentis, Samumed, Vericel, and ZKR Orthopedics; research support and/or royalties from Active Implants, Arthrex, Biopoly, Episurf, Fidia, JRF Ortho, Moximed, Novartis, Organogenesis, Samumed, Springer, Theime Medical Publishers, Vericel, and ZimmerBiomet; and stock or stock options from Ortho Regenerative Tech and MedShape. Dr. Conaghan has received consulting fees from AbbVie, Contura, Eli Lilly, EMD Serono, Flexion Therapeutics, Galapagos, Gilead, Pfizer, and Novartis.

Compliance with Ethics Guidelines. The research within this clinical trial was conducted in accordance with the Declaration of Helsinki, the International Council for Harmonisation Good Clinical Practice Guidelines, and all applicable regulations. All sites conducted the trial under institutional review board approval. All participants provided written informed consent prior to engaging in any study-related procedures.

Data Availability. Data will be made available upon reasonable request on a case-by-case basis as they are part of an ongoing clinical investigation.

Open Access. This article is licensed under a Creative Commons Attribution-NonCommercial 4.0 International License, which permits any non-commercial use, sharing, adaptation, distribution and reproduction in any medium or format, as long as you give appropriate credit to the original author(s) and the source, provide a link to the Creative Commons licence, and indicate if changes were made. The images or other third party material in this article are included in the article's Creative Commons licence, unless indicated otherwise in a credit line to the material. If material is not included in the article's Creative Commons licence and your intended use is not permitted by statutory regulation or exceeds the permitted use, you will need to obtain permission directly from the copyright holder. To view a copy of this licence, visit http://creativecommons.org/licenses/by$\mathrm{nc} / 4.0 /$.

\section{REFERENCES}

1. Cross M, Smith E, Hoy D, Nolte S, Ackerman I, Fransen M, et al. The global burden of hip and knee osteoarthritis: estimates from the Global Burden of Disease 2010 study. Ann Rheum Dis. 2014;73: 1323-30.

2. Dworkin RH, Turk DC, Wyrwich KW, Beaton D, Cleeland CS, Farrar JT, et al. Interpreting the clinical importance of treatment outcomes in chronic pain clinical trials: IMMPACT recommendations. J Pain. 2008;9:105-21.

3. Tubach F, Ravaud P, Baron G, Falissard B, Logeart I, Bellamy N, et al. Evaluation of clinically relevant states in patient reported outcomes in knee and hip osteoarthritis: the patient acceptable symptom state. Ann Rheum Dis. 2005;64:34-7.

4. Smith SM, Dworkin RH, Turk DC, McDermott MP, Eccleston C, Farrar JT, et al. Interpretation of chronic pain clinical trial outcomes: IMMPACT 
recommended considerations. Pain. 2020;161: 2446-61.

5. Pham T, Der Heijde D, Van LM, Altman RD, Anderson JJ, Bellamy N, et al. Outcome variables for osteoarthritis clinical trials: THE OMERACT-OARSI set of responder criteria. J Rheumatol. 2003;30: $1648-58$.

6. Yazici Y, McAlindon TE, Gibofsky A, Lane NE, Clauw D, Jones $\mathrm{M}$, et al. Lorecivivint, a novel intraarticular CDC-like kinase 2 and dual-specificity tyrosine phosphorylation-regulated kinase $1 \mathrm{~A}$ inhibitor and Wnt pathway modulator for the treatment of knee osteoarthritis: a phase II randomized trial. Arthritis Rheumatol. 2020;72: 1694-706.

7. Deshmukh V, O'Green AL, Bossard C, Seo T, Lamangan L, Ibanez $\mathrm{M}$, et al. Modulation of the Wnt pathway through inhibition of CLK2 and DYRK1A by lorecivivint as a novel, potentially disease-modifying approach for knee osteoarthritis treatment. Osteoarthr Cartil. 2019;27:1347-60.

8. Yazici Y, Mcalindon TE, Gibofsky A, Lane NE, Lattermann C, Skrepnik N, et al. A phase $2 b$ randomized trial of lorecivivint, a novel intra-articular CLK2/DYRK1A inhibitor and Wnt pathway modulator for knee osteoarthritis. Osteoarthr Cartil. 2021;29:654-66.

9. Bellamy N, Bell MJ, Goldsmith $\mathrm{CH}$, Pericak D, Walker V, Raynauld J-P, et al. The effectiveness of hylan G-F 20 in patients with knee osteoarthritis: an application of two sets of response criteria developed by the OARSI and one set developed by OMERACT-OARSI. Osteoarthr Cartil. 2005;13: 104-10.

10. Conaghan PG, Cohen SB, Berenbaum F, Lufkin J, Johnson JR, Bodick N. Brief report: a phase IIb trial of a novel extended-release microsphere formulation of triamcinolone acetonide for intraarticular injection in knee osteoarthritis. Arthritis Rheumatol. 2018;70:204-11.

11. Hochberg MC, Wohlreich M, Gaynor P, Hanna S, Risser R. Clinically relevant outcomes based on analysis of pooled data from 2 trials of duloxetine in patients with knee osteoarthritis. J Rheumatol. 2012;39:352-8.

12. Dakin P, DiMartino SJ, Gao H, Maloney J, Kivitz AJ, Schnitzer TJ, et al. The efficacy, tolerability, and joint safety of fasinumab in osteoarthritis pain: a phase IIb/III double-blind, placebo-controlled, randomized clinical trial. Arthritis Rheumatol. 2019;71:1824-34.

13. Schnitzer TJ, Bello AE, West CR, Pixton GC, Tive L. Response to Tanezumab, as assessed by outcome measures in rheumatology-osteoarthritis research society international criteria, in patients with osteoarthritis of the knee or hip. Osteoarthr Cartil. 2018;26:S299.

14. Previtali D, Merli G, Di Laura FG, Candrian C, Zaffagnini S, Filardo G. The long-lasting effects of "placebo injections" in knee osteoarthritis: a metaanalysis. Cartilage. 2020. https://doi.org/10.1177/ 1947603520906597. 